\title{
AMENDMENTS
}

\section{Publisher Correction: Resolved-sideband Raman cooling of an optical phonon in semiconductor materials}

Jun Zhang, Qing Zhang, Xingzhi Wang, Leong Chuan Kwek and Qihua Xiong

Correction to: Nature Photonics https://doi.org/10.1038/nphoton.2016.122, published online 4 July 2016.

In this Article, the equation following the sentence "The extra damping introduced can be written as:" was incorrect and should have appeared as shown below:

$$
\Gamma_{\mathrm{net}}=g\left(\frac{\kappa_{\mathrm{ex}}}{\kappa_{\mathrm{ex}}^{2} / 4+\left(\Delta+\Omega_{\mathrm{LOP}}\right)^{2}}-\frac{\kappa_{\mathrm{ex}}}{\kappa_{\mathrm{ex}}^{2} / 4+\left(\Delta-\Omega_{\mathrm{LOP}}\right)^{2}}\right)
$$

Published online: 25 April 2019

https://doi.org/10.1038/s41566-019-0440-4

\section{Publisher Correction: Biophotonics: Plasmon-enabled disinfection}

Noriaki Horiuchi

Correction to: Nature Photonics https://doi.org/10.1038/s41566-019-0429-z, published online 24 April 2019.

In the version of this News \& Views article originally published, the final digit was missing from the doi to the linked article: 'https://doi. org/10.1021/acs.nanolett.9b0018' should have read 'https://doi.org/10.1021/acs.nanolett.9b00187'. This has now been corrected in the online versions. 